\title{
Présence de L-lactate dans les sécrétions digestives du lapin
}

\author{
Michèle VERNAY \\ Laboratoire de Physiologie de la Digestion et de la Nutrition \\ 2, rue F.-Magendie, 31400 Toulouse
}

\begin{abstract}
Résumé
La présence de L-lactate est recherchée dans les sécrétions digestives (salivaire, gastrique, biliaire, pancréatique, intestinale, colique) du lapin domestique nourri ad libitum. Du L-lactate est présent dans tous les sucs étudiés; cependant le taux de cet acide organique est nettement plus élevé dans la bile $(2,8 \pm 0,3 \mathrm{mM})$.
\end{abstract}

Mots clés : lapin, lactate endogène, sécrétions digestives, lactacidémie.

\section{Introduction}

Contrairement à de nombreuses espèces : rat (RÉMÉsy \& Demigné, 1976), porc (WIRTZ \& BickHARDT, 1977), poney (ARGENZIo et al., 1974), la fermentation lactique dans le matériel digestif du lapin est toujours très discrète (VERNAY, 1985), ce qui va dans le sens de l'absence d'une flore de type lactobacille (FonTy, 1974). Cependant des taux non négligeables de lactate sont retrouvés dans le bol alimentaire, notamment dans l'intestin grêle (AlEXANDER \& ChOWdHury, 1958) et dans le gros intestin (MARTy, RAYNAUD \& CARLES, 1973). Cet ensemble de faits nous a conduit à rechercher la présence de L-lactate dans les différentes sécrétions digestives du lapin.

\section{Matériel et méthodes}

Au total 27 lapins de race commune et d'un poids moyen de $2,5 \mathrm{~kg}$ sont utilisés. Ils sont nourris ad libitum de luzerne et d'avoine. Sur 8 animaux anesthésiés au pentobarbital $(20 \mathrm{mg} / \mathrm{kg})$ nous prélevons de la salive à l'aide d'une micropipette ainsi que les sécrétions pancréatique et biliaire, après cathétérisation des canaux respectifs. La confection d'anses chroniques de type Thiry-Vella permet de recueillir les sécrétions intestinale (4 lapins) et colique (10 lapins). Le suc gastrique de 5 sujets 
différents est obtenu à partir de poches établies selon la technique de BEAuville \& RaYNAud (1968). Du sang artériel est recueilli, sur héparine, au niveau de l'oreille. Les différents échantillons sont prélevés le matin entre 9 et 10 heures, puis centrifugés $(2 \mathrm{mn}$ à $10000 \mathrm{~g})$. La teneur en L-lactate est déterminée par l'action de la L-lactate déshydrogénase (EC 1.1.1.27) de Boerhinger en présence de NAD, selon le procédé de HoHorst (1965).

\section{Résultats et discussion}

L'analyse des résultats consignés dans le tableau montre qu'effectivement du L-lactate est présent dans toutes les sécrétions recueillies. Le taux de cet acide organique est toutefois plus élevé dans la bile, ce qui est en accord avec les travaux de LE BARs et al. (1969). Ces auteurs rapportent l'existence d'un cycle entéro-hépatique pour le lactate chez le lapin. La présence de L-lactate dans les sécrétions : gastrique, intestinale ou colique est très certainement liée à l'activité métabolique de la muqueuse intestinale. En effet il a été montré, in vitro et in vivo, que le tissu digestif est capable de produire du lactate à partir de substrats tels que le glucose (Hanson \& Parsons, 1976) et les acides gras volatils (Worbe \& Mottaz, 1979 ; Marty \& Vernay, 1984). Le lactate synthétisé peut être provisoirement stocké dans les cellules de l'épithélium gastro-intestinal et/ou éliminé soit dans la lumière du tube digestif, soit dans la circulation sanguine. Les sécrétions digestives contribuent donc à enrichir le digesta en L-lactate.

\section{Tableau 1}

Concentrations ( $m M, m g / 100 \mathrm{ml}$ ) du L-lactate dans les sécrétions digeștives et dans le plasma artériel correspondant. Lactate concentrations ( $\mathrm{mM}, \mathrm{mg} / 100 \mathrm{ml}$ ) in the digestive secretions and in the corresponding arterial plasma.

\begin{tabular}{|c|c|c|c|c|}
\hline \multirow{2}{*}{$\begin{array}{l}\text { Sécrétions } \\
\text { Secretions }\end{array}$} & \multicolumn{2}{|c|}{$\begin{array}{l}\text { L-lactate } \\
\text { L-lactate }\end{array}$} & \multicolumn{2}{|c|}{$\begin{array}{l}\text { Lactacidémie } \\
\text { Lactacidemia }\end{array}$} \\
\hline & $\mathrm{mM}$ & $\mathrm{mg} / 100 \mathrm{ml}$ & $\mathrm{mM}$ & $\mathrm{mg} / 100 \mathrm{ml}$ \\
\hline $\begin{array}{l}\text { Salivaire }(8) \quad \ldots \ldots \ldots \\
\text { Salivary }(8)\end{array}$ & $1,2 \pm 0,2$ & $10,4 \pm 1,6$ & $1,6 \pm 0,2$ & $14,2 \pm 1,8$ \\
\hline $\begin{array}{l}\text { Gastrique }(10) \ldots \ldots \\
\text { Gastric }(10)\end{array}$ & $0,7 \pm 0,1$ & $6,6 \pm 0,5$ & $1,8 \pm 0,3$ & $16,9 \pm 2,6$ \\
\hline $\begin{array}{l}\text { Intestinale (7) } \ldots \ldots \\
\text { Intestinal (7) }\end{array}$ & $0,9 \pm 0,4$ & $8,8 \pm 3,3$ & $2,1 \pm 0,1$ & $18,5 \pm 0,9$ \\
\hline $\begin{array}{l}\text { Biliaire (16) } \ldots \cdots \cdots \\
\text { Biliary }(16)\end{array}$ & $2,8 \pm 0,3$ & $24,7 \pm 1,7$ & $1,6 \pm 0,2$ & $14,2 \pm 1,8$ \\
\hline $\begin{array}{l}\text { Pancréatique (6) } \ldots \\
\text { Pancreatic (6) }\end{array}$ & $1,8 \pm 0,2$ & $16,6 \pm 2,1$ & $1,6 \pm 0,2$ & $14,2 \pm 1,8$ \\
\hline $\begin{array}{l}\text { Colique }(22) \ldots \ldots \cdots \\
\text { Colic }(22)\end{array}$ & $0,5 \pm 0,2$ & $4,6 \pm 1,1$ & $2,2 \pm 0,1$ & $19,6 \pm 0,9$ \\
\hline
\end{tabular}




\section{Summary \\ Evaluation of L-lactate in digestive secretions of the rabbit}

L-lactate was evaluated in the digestive secretions (salivary, gastric, biliary, pancreatic, intestinal, colic) of the domestic rabbit provided with oats and lucerne ad libitum. It appeared that L-lactate was present in the different fluids studied, but a high level was found for the bile $(2.8 \pm 0.3 \mathrm{mM})$. This high value can be explained by the existence of a lactate entero-hepatic cycle; meanwhile the endogenous lactate production in the gut secretion was certainly linked to the metabolism of the tissue.

Key words : rabbit, endogenous lactate production, digestive secretions, lactacidemia.

\section{Références bibliographiques}

Alexander F., Chowdhury A.K., 1958. Digestion in the rabbit's stomach. Br. J. Nutr., 12, 65-73.

Argenzio R.A., Southworth M., Stevens C.E., 1974. Sites of organic acids production and absorption in the gastrointestinal tract of the pig. Am. J. Physiol., 228, 454-460.

Beauville M., Raynaud P., 1968. Nouvelle technique de confection d'une poche gastrique isolée chez le lapin. C.R. Acad. Sci., 267, 1153-1156.

FonTY G., 1974. Etude qualitative et quantitative de la microflore digestive du lapin holoxénique. Thèse $3^{\mathrm{e}}$ cycle, Université de Clermont-Ferrand II, 65-80.

HANSON P.J., PARSONS D.S., 1976. The utilization of glucose and production of lactate by in vitro preparations of rat small intestine : effects of vascular perfusion. J. Physiol. (G.B.), 255, 775-795.

HoHORst H.J., 1965. In : «Methods of enzymatic analysis», BERGMEYer H.U., 266-277, Acad. Press, London and New York.

Le Bars, Mahboub S., Demaux G., 1969. Existence d'un cycle entéro-hépatique de l'acide lactique chez le lapin. Journées d'études sur le lapin, Alfort, 1-12, A.S.F.C. Paris ed.

Marty J., Raynaud P., Carles J., 1973. Les acides aminés et les acides carboxyliques dans le caecum du lapin. Ann. Biol. Anim. Biochim. Biophys., 13, 429-451.

Marty J., Vernay M., 1984. Absorption and metabolism of the volatile fatty acids in the hind-gut of the rabbit. Br. J. Nutr., 52, 265-277.

RÉmésy C., Demgané C., 1976. Partition and absorption of volatile fatty acids in the alimentary canal of the rat. Ann. Rech. Vétér., 7, 39-55.

VERNAY M., 1985. L'absorption et le devenir des acides gras volatils digestifs chez le lapin, en relation avec la dualité de l'émission fécole. Thèse de doctorat d'Etat, Université Paul Sabatier, Toulouse III, 53-95.

Wirtz A., BickHardT K., 1977. Zum Ruhe. Lactatstoffwechsel bei nüchternen und gefütterten. Schweinen. Z. Tierphysiol. Tierernähr. Futtermittelkde, 39, 146-156.

Worbe J.J., MotTaz P., 1979. Synthèse d'acide lactique par le caecum de rat au cours du transfert des acides gras volatils. Ann. Biol. Anim. Biochim. Biophys., 19, 723-728. 